\title{
LANDSCHAFT UND PFLANZENVEREIN
}

\author{
Mit besonderer Berücksichtigung russischer Forschungen ${ }^{1}$ \\ Von Constantin v. Regel
}

Mit 3 Abbildungen

Die Pflanzensoziologie, Phytosoziologie oder Phytocoenologie, steckt wie manche junge Zweige der biologischen Wissenschaften noch im Zustand des Ringens um die richtigen Methoden und um Begriffs- und Theorienbildung. Das Methodologische spielt deshalb in ihr eine unverhältnismäßig große Rolle, und methodologische Fragen nehmen einen bedeutenden Teil ihres Schrifttums ein. Dies gilt besonders auch für die Begriffsbestimmung und Klassifikation der Pflanzenvereine (Pflanzengesellschaften oder Phytocoenosen), bei der bisher keine einheitliche Auffassung erzielt wurde ${ }^{2}$.

So stellen z. B. Morozow (1926) und Sukatschew $(1931,1932,1938)$ die Grundeinheit der Waldvereine, die Waldassoziation, dem Waldtypus gleich. Für andere sind Waldverein und Waldtypus verschiedene Dinge. In einer Klassifikation der Wälder (Korchagrn 1946) lassen sich entweder alle ökologischen Faktoren: Boden, Wasser, Luft usw. einbeziehen, oder aber die Vegetation wird ausschließlich verwendet; der Standort, der ja nicht direkt zur Vegetation gehört, kein unmittelbarer Vegetationsbestandteil ist, bleibt unberücksichtigt.

Auch bei der Klassifikation der Wiesen, Heiden, Moore usw. bestehen Widersprüche, wie aus zahlreichen Vegetationsbeschreibungen hervorgeht. Wir finden in der ausgezeichneten, wenn auch, weil auf einem einzigen Merkmal, der Physiognomie, beruhenden künstlichen (besser: partiellen) Klassifikation von BROCKMANN-JEROSCH und RüBEL (1912) und dann bei RübeL (1930) neben den Duriprata (Hartwiesen) bzw. Duriherbosa die Sphagniprata bzw. Sphagniherbosa (Hochmoore). Jene gründen auf einem vegetationsphysiognomischen Merkmal, sind also eine Zusammenfassung von Pflanzenvereinen; letztere dagegen sind Landschaften, die aus verschiedensten, physiognomisch ungleichen Pflanzenvereinen bestehen. Analoge Überlegungen gelten hinsichtlich der Heiden, Steppen usw.

Die begrifflichen Differenzen beruhen vor allem auf der Tatsache, daß die Termin. Pflanzenverein und Landschaft von vielen Botanikern vermengt werden, und ihre Überwindung hat auf deren klare Auseinanderhaltung aufzubauen. Hierzu soll im folgenden beigetragen werden, wobei die russische Forschung besonders berücksichtigt wird, weil ihre zahlreichen Beiträge zum Problem, bisher kaum gewürdigt, wesentlich erscheinen.

\section{Der Begriff Landschaft}

Eine unübersehbare Literatur behandelt die Landschaft und ihre begriffliche Fixierung. Aus ihr können nur wenige Definitionen herausgegriffen werden, die dem in der Praxis hauptsächlich verwendeten Begriff am meisten entsprechen.

Passarge (1916) nennt natürliche Landschaft «ein Gebiet, das möglichst nach Klima, Pflanzendecke, Oberflächengestaltung, Bewässerung, geologischem Bau und Boden eine Einheit darstellt. Im allgemeinen fallen nicht alle genannten Erscheinungen zusammen; einige müssen aber zu einer Einheit zusammentreten, wenn eine Landschaft entstehen soll». SEMJonow-TJAN-SCHANSki (1928) sagt, daß der Geograph zur wissenschaftlich objektiven Erfassung der Landschaft alles Klare und Typische auszuwählen habe und alle Erscheinungen im weitesten Umfang, dabei auch Gerüche (z. B. den Harzgeruch der finnischen Wälder) und Töne (z. B. die Vogelstimmen im Wald) mit berücksichtigen müsse. "Landschaft», betont BERG (1930) "sind gesetzmäßig sich wiederholende Gruppierungen nicht nur

1 Die Arbeit wurde im Geobotanischen Institut Rübel in Zürich ausgeführt, dessen Leiter, Herrn Direktor Dr. W. LüDI, der Verfasser hiermit seinen Dank ausspricht. Ferner ist er Herrn Pd. Dr. E. WINKLER am Geographischen Institut der ETH für die vielen Hinweise auf geographischem Gebiet und der Leitung dieses Institutes, Herrn Prof. Dr. H. Gutersohn, für die Möglichkeit der Benutzung von dessen Bibliothek zu Dank verpflichtet.

2 Pflanzenverein, Pflanzengesellschaft und Phytocoenose bedeuten ein und dasselbe. Die weiter unten zu besprechende Assoziation ist die grundlegende von den Pflanzensoziologen gebrauchte Einheit des Pflanzenvereins, der "Art» der systematischen Botanik analog, deren Begrenzung jedoch oft rein subjektiv erfolgt. Eine noch kleinere Einheit ist die Soziation, die der Unterart der Systematiker entspricht. 
der Formen des Reliefs, wie sie z. B. PENCK (1894) als für die Landschaft charakteristisch ansieht, sondem auch anderer Erscheinungen und Gegenstände der Oberfläche.» Man müsse im Auge behalten, $\mathrm{daB}$ die geographische Landschaft eine solche Gesamtheit der Gruppierung der Gegenstände und Erscheinungen sei, in der die Merkmale des Reliefs, des Klimas, der Gewässer, des Bodens, der Vegetationsdecke und der Tierwelt sowie auch die Tätigkeit des Menschen sich zu einem harmonischen Ganzen vereinigen, das sich in typischer Weise in andern Teilen der Erdoberfläche wiederhole. Die Landschaft erscheint danach als eine Art Vergesellschaftung höheren Grades, die einerseits die Gesellschaften von Organismen (Biocoenosen), d. h. von Pflanzen (Phytocoenosen), vonTieren (Zoocoenosen) und von Menschen (Anthropocoenosen), anderseits aber auch die Komplexe der anorganischen Erscheinungen, der Erdrinde, des Wassers und Klimas in sich schließt, ebenso die Böden, die ein Produkt der organischen Welt und der anorganischen Teile der Erdrinde sind. In jeder Landschaft erkennt man nach BERG eine vollständige Anpassung aller ihrer Elemente aneinander. Jede Landschaft ist also ein Organismus, in dem die einzelnen Teile auf das Ganze und dieses wieder auf die einzelnen Teile wirken. Wenn wir den einen Teil verändern, so verändern wir auch das Ganze. Diese letztere Ansicht stimmt mit derjenigen zahlreicher anderer Geographen überein, wie z. B. MURis (1930), LaUtensach (1938) u. a. HARTSHORNE (1939) versteht unter einer geographischen Landschaft einen Teil der Erdoberfläche, in welchem Atmosphäre, Vegetation, Boden, Schnee, Eis oder Wasser und die vom Menschen geschaffenen Gegenstände in unmittelbarem Zusammenhang stehen. Nach WINKLER (1946) schließlich repräsentiert sie den Korrelationseffekt von Litho-Hydro-Atmo- und Biosphäre der Erde.

Landschaft ist somit in mehr oder weniger allgemeiner Übereinstimmung der Geographen als ein Stück der Erdhülle zu definieren, das erstens aus Boden, Gewässern, Lufthülle, Vegetation, Tierwelt, Mensch und Menschenwerk besteht und zweitens in deren Zusammenwirken eine (harmonische) Einheit im Sinne eines Wirkungszusammenhangs darstellt. Ihr Wesen liegt somit in erster Linie in der Gesamtkorrelierung der terrestrischen Seinssphären.

Damit gilt zugleich, daß die einzelnen Komponenten sich zwar isolieren lassen und wir mit einzelnen von ihnen die Landschaft zu kennzeichnen versuchen können, dann jedoch immer einen nur unvollständigen Begriff von ihr erhalten.

Wenn wir zum Beispiel eine Landschaft mit der Vegetation, wie schon A. v. Humbordt und jüngst besonders J. SCHMITHüsEN (1942) betonten, einem ihrer zweifellos zumeist wesentlichen Elemente, allein charakterisieren, so gewinnen wir in der Regel ein anschauliches Bild von ihr, auch wenn die anderen Merkmale, wic Boden und Klima, nicht berüicksichtigt sind. Beschreiben wir zum Beispiel die baumlose Tundra auf Grund der Vegetation allein, so erhalten wir einen vollständigeren Landschaftseindruck, als wenn wir sie nur auf Grund des Klimas allein oder des Bodens oder der Tierwelt beschreiben würden. Eine völlige Erfassung ermöglicht sich aber damit nicht.

Die Vegetation ist indes nichts stets das prägnanteste Landschaftselement. In einer Stadtlandschaft treten zam Beispiel die Bauten des Menschen in den Vordergrund, und der Einfluß des Menschen auf die Landschaft wird um so größer, je mehr sich die ursprüngliche Naturlandschaft in eine Wirtschaftsund Kulturlandschaft umwandelt ${ }^{3}$. In der UdSSR wurden demgemäß Abgrenzungen von Landschaften auf Grund wirtschaftlicher Erscheinungen vorgenommen. Ihr ganzes Gebiet, aber auch einzelne Teilgebiete sind rayonniert worden. Zum Beispiel teilte Suworow (1932) Weißrußland in einen LeinMilch-Rayon, einen Milch-Lein-Rayon, einen Rayon mit geringem Anbau von Lein und einen Rayon mit Schweinezucht, also in vier Gebiete, die durch landwirtschaftliche Momente gekennzeichnet sind. Eine noch eigenartigere Gliederung Weißrußlands nach Kartoffelkrankheiten gaben DarashkiN und RawDo. Bei der Untersuchung der im Mittelmeergebiet verbreiteten Arten der Gattung Phillyrea stellte ich 1949 fest, daß die Ostmediterranis durch das Vorkommen einer Form der Phillyrea charakterisiert werden könne, deren Blätter ein bestimmtes Verhältnis von Länge zu Breite aufweisen.

Solche Beispiele ließen sich auch aus der Fachliteratur anderer Länder zahlreiche heranziehen. Sie erweisen alle, daß einzelne Landschaftselemente und -elementgruppen zwar oft eine weitgehende Landschaftscharakteristik ermöglichen, daß im Grunde jedoch nur eine Berücksichtigung des Gesamtbestandes eine objektive Fixierung erlaubt.

\section{Landschaft und Pflanzenverein}

Die aus Pflanzenvereinen bestehende Vegetationsdecke repräsentiert demzufolge in jedem Fall einen Bestandteil, ein Element der Landschaft; die pflanzengeographische Provinz, wie sie von den Pflanzengeographen beschrieben wird, ist nichts anderes als

3 Die Landschaft ist daher auch nicht etwas Beständiges, Statisches. Sie ist einem ständigen Wandel unterworfen. WinkLER $(1933,1937)$ definierte sie deshalb als sachlich-räumlich-zeitliches Gebilde. Und gerade die Zeitlichkeit der Landschaft, ihr Wandel ist zu berücksichtigen, wenn sie konkret erfaßt werden soll. Dies betont u. a. auch Sorschawa (1947). 
Abb. 1. Gebirgswaldund Weidelandschaft am Oxya, oberhalb Muzaki (Thessalien.) ImVordergrund Getreidefelder, in der Mitte Buchengehölz auf Flysch, im höhern Hintergrund auf Kalk Tannengehölz und Karst. Hier tritt der Unterschied von Vegetation und Landschaft klar hervor. Photo: C.v. REGEL. Aus Botanische Jahrbücher, 73, 1943. E. Schweizerbartsche Verlagsbuchhdl., Stuttgart.

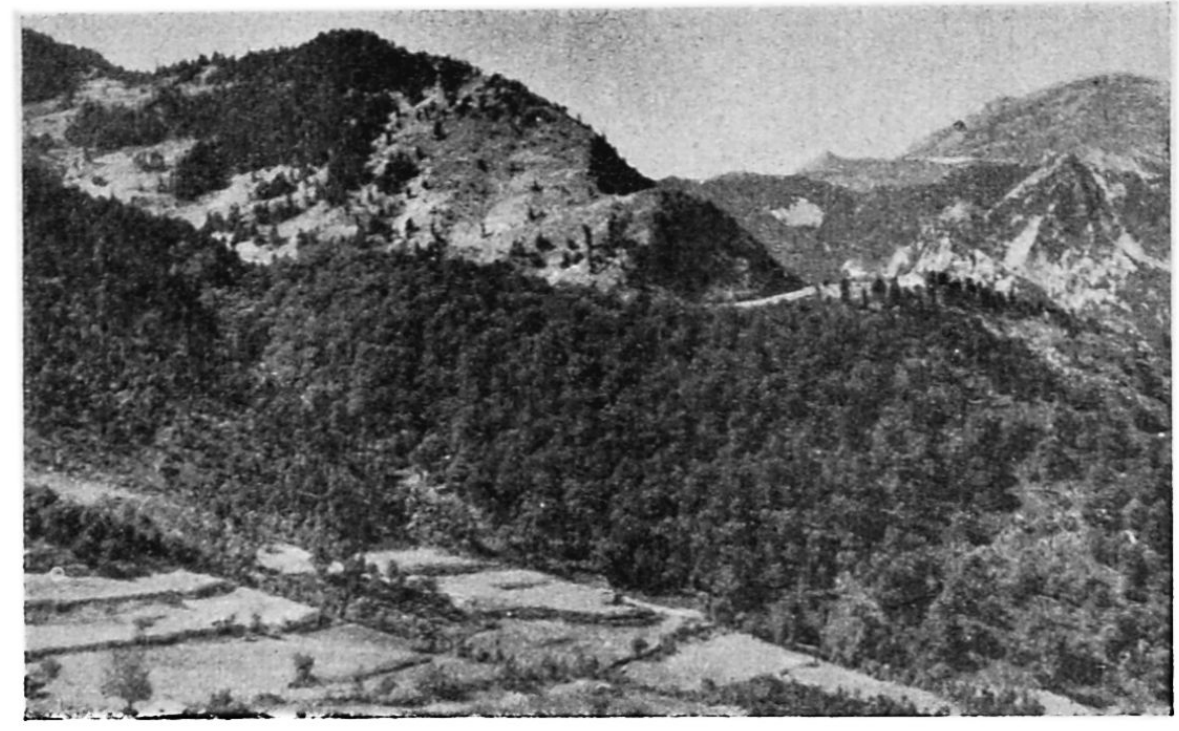

deren botanische Charakteristik (Zinserling 1934, Regel 1941). Landschaft und Pflanzendecke sind somit nie als identische Gebilde aufzufassen. Doch kann die Landschaft wohl ihre Charakterisierung nach dem vorherrschenden Pflanzenverein erhalten und darnach benannt werden.

$\mathrm{Da}$ der Wald als eine Landschaft aufzufassen ist, erkannte schon KrüDENER (1926), der den Waldtypus die kleinste natürliche Landschaftseinheit, einen Mikrolandschaftstypus, nannte. Morozow (1928) behandelt in seiner Lehre vom Walde den Begriff der Landschaft in deren Beziehungen zum Walde, indem er sagt, der Wald sei eine geographische Erscheinung. Er ist "ein Naturelement und gleich den Steppen, Wüsten und Tundren ein Teil der Landschaft, folglich ein Teil der Erdoberfäche, die kraft ihrer bestimmten biologischen Eigenschaften von entsprechenden Waldgemeinschaften bedeckt ist».

Die Umwelt bedingt die Zusammensetzung des Waldes. Die Vergesellschaftungen, die die Holzarten miteinander eingehen, werden bedingt durch die äußeren Bedingungen einerseits, die inneren Eigenschaften der Holzgewächse anderseits und - oft - drittens durch Einwirkung des Menschen. "Der Wald ist eine soziale und auch eine geographische Erscheinung", sagt Morozow. Diese Auffassung des Waldes als einer Landschaft liegt, ohne daß es ausgesprochen wird, allen Klassifikationsversuchen der Wälder zugrunde, die auf den ökologischen Faktoren beruhen. Nach Sukatschew (1942) ist der Wald schlechthin einer Geocoenose, d. h. einer Elementarlandschaft, gleichzustellen. Die Waldassoziation dagegen ist nach ihm die rein botanische, pflanzensoziologische Klassifikationseinheit der Vegetationsdecke. Die Waldtypen Sukatschews (1931, 1932, 1938), Krüdeners (1916, 1926), Cajanders (1909), die Waldtypen, die Regel (1941) als Assoziationskomplexe auf der Halbinsel Kola beschreibt, sind nichts anderes als Landschaften, in denen die aus Gehölzen gebildeten Vereine, die wir gewöhnlich Wälder nennen, die aber nach RüBEL (1930) zu den Lignosa gehören, vorherrschen.

Die von REGEL auf der Halbinsel Kola beschriebenen Komplexe lassen sich durch verschiedene Merkmale charakterisieren, durch den Boden und auch durch eine bestimmte Breite der Jahresringe, einen bestimmten Jahresringetypus der vorherrschenden Holzart. Die Pflanzendecke dieser Komplexe ist nicht homogen, sondern setzt sich aus verschiedenen Pflanzenvereinen, Gehölzen, Zwergsträuchern, Flechten usw. zusammen. Dementsprechend ist natürlich die Zone der nördlichen Nadelwälder Eurasiens, die sogenannte Taiga, eine Landschaft, jedoch höheren Grades. Sie besteht aus zahlreichen, physiognomisch verschiedenen Pflanzenvereinen: Wäldern, Sumpfen, Mooren, Wiesen usw. Sie ist also zunächst eine Vegetationsregion im Sinne von DU RIETZ, ein Phytocoenosenkomplex. Dazu gehören aber Boden, Klima, Tierwelt und, wenn auch mit geringerem Wirkungsgrad, der Mensch. Die Taiga zerfällt in kleinere Landschaftseinheiten, wie z. B. das Nadelwaldgebiet Fennoskandiens, des Nordostens von Rußland, Westsibiriens usw., die sich in noch kleinere Einheiten mit nicht selten lokalen Benennungen untergliedern, die KRüDENER nicht mehr lokal anwendet.

Aus allem resultiert, daß «Wald» nicht ein Pflanzenverein, sondern ein Landschaftstyp ist, in dem die Bäume oder auch bestimmte Baumvereine vorherrschen, von denen er daher ihren Namen erhält. Die pflanzensoziologische Benennung des vorherrschenden Pflanzenvereins wäre "Lignosa", "Gehölz» oder "Waldverein» (-gesellschaft ». Konsequenterweise dürfte in der Phytosoziologie nur von Nadelgehölzen, Laubgehölzen usw. gesprochen werden; die mit dem Ausdruck Wald zusammenhängenden Begriffe dagegen müßten der Landschaftskunde reserviert bleiben, wobei in dieser in der Gegenwart - in einem gewissen Gegensatz dazu - freilich der Usus besteht, dafür den Terminus Waldlandschaft zu verwenden. 
Auch die Steppe ist, worauf GradmanN (1916) und später Regel (1939) hinwiesen, eine Landschaft. Sie ist zudem regional aufzufassen; der Name "Steppe» darf nicht auf andere ähnliche Gebiete übertragen werden, oder wenn man dies doch tut, so nur mit einem bestimmten Korrektiv. Es lassen sich nach RüBEL (1930) im Gebrauch des Wortes Steppe zwei Hauptrichtungen unterscheiden. Die eine, im deutschen und französischen Sprachgebiet allgemein verbreitete und auch von den Geographen verwendete, schließt alle baumlosen Trockengebiete ein, von der mäßig trockenen Wiese bis zur Wüste mit stark wechselnder Ókologie, wobei ENGLER noch weiter geht und lichte Wälder dazu rechnet ("Obstgartensteppe»). Die andere Richtung beschränkt die Steppe auf ihre ursprüngliche Bedeutung der Hartgraswiesen Südrußlands. Trotzdem aber RüBEL meint, daß diese letztere botanisch wohl richtige Ansicht wegen der Verbrauchtheit des Wortes Steppe nicht mehr durchführbar scheine, wird hier wie früher (REGEL 1939) diese zweite Ansicht vertreten. Denn die Steppe ist eine Landschaft, zu deren Hauptmerkmal das Fehlen der Bäume, die Schwarzerde und ein charakteristisches Klima gehören, eine Auffassung, die von manchen russischen Pflanzengeographen, wie KrasNOw (1924), KorshINSKI (1901); KeLLER (1923), BUSCH (1936) geteilt wird. Nicht nur eine Reihe botanischer Merkmale charakterisieren also die Landschaft «Steppe», sondern noch andere, von denen wir nur einige hervorhoben. Die Landschaft «Steppe» umfaßt zudem verschiedene Pflanzenvereine, von denen die aus Gräsern bestehenden und an Trockenheit angepaßten am meisten verbreitet sind. Indes enthält sie auch Sümpfe und gewisse Waldformen. Demgegenüber ist, was Brockmann-Jerosch, Rübel (1912) Siccideserta und Rübel (1930) Duriherbosa nannten, nicht Steppe, sondern nur eine Benennung für Pflanzenvereine von bestimmtem physiognomischen Tapus, die auch im Gebiete der Steppe vorkommen und dieser ihr charakteristisches Aussehen geben. Diese Vereine sind jedoch auch in der Prärie verbreitet, die keine Steppe, sondern eine ihr im westlichen Teil der nördlichen Halbkugel äquivalente, aber von dieser auch in wesentlichen Merkmalen verschiedene Landschaft ist. REGEL (1939) sah in Marokko wiesenartige Vereine, die der Wiesensteppe in Rußland an die Seite zu stellen sind, sich jedoch von ihr floristisch stark unterscheiden. Man müßte sie marokkanische Wiesensteppe nennen oder einen anderen lokalen Ausdruck dafür benutzen, nicht aber den in Rußland gebräuchlichen Namen "Wiesensteppe» oder "Waldsteppe», die beide ineinander übergehen und einen besonderen Landschaftstypus darstellen, der an der Grenze zwischen den Landschaftstypen "Wald» und "Steppe» liegt. Die diese Landschaft charakterisierenden Pflanzenvereine sind "Aestilignosa» und bestimmte Typen der "Sempervirentiherbosa", um die von RüBEL eingeführten physiognomischen Ausdrücke zu verwenden.

Das ständige Verwechseln von Landschaft und Pflanzenverein hat auch in der Pflanzengeographie zu Ungenauigkeiten geführt. So spricht man vom pontischen Element, worunter man Pflanzen versteht, deren Hauptverbreitung in der Steppe liegt. Dabei wird immer wieder vergessen, daß die Steppe kein Pflanzenverein, sondern eine Landschaft ist, in der es Duriherbosa mit Trockenheit liebenden Pflanzen gibt, aber auch andere Pflanzenvereine, die nicht immer auf trockenen Böden stocken. Daher brauchen die pontischen Pflanzen nicht immer xerophile, an Trockenheit gewöhnte Pflanzen zu sein. Im Gegenteil existieren auch solche auf feuchten Böden, die mesophilen Charakter tragen'.

Der Steppe ähnlich ist die waldlose, nördlich der polaren Waldgrenze sich erstreckende Landschaft der Tundra in Asien und Europa (Gorodkow 1935, Regel 1933 und 1941). Auch sie ist kein Pflanzenverein; die Zone der Tundra zerfällt in zahlreiche kleinere und kleinste Unterabteilungen, in kleinere Einheiten, kleinere Naturkomplexe. Wie REGEL und GoRoDKow zeigen, gibt es Tundrawälder, Wiesen, Moore, Gestrüppe, Kältewüsten und andere Pflanzenvereine in der Tundra. Wenn die Vegetation der Tundra auch manche Ähnlichkeiten mit der alpinen Stufe der Gebirge aufweist, so sind Tundra und alpine Stufe nicht dasselbe, und es wäre falsch, wie einige tun, von Gebirgstundren zu sprechen.

Auch die Dünen können als Landschaften angesehen werden, jedoch als azonale, nicht klimatisch bedingte, denen die Mobilideserta (RüBEL 1930) den charakteristischen Stempel aufdrücken. Der Name Dünenland schaften wäre indes vielleicht zutreffender. REGEL versuchte (1928) auf genetischer Grundlage eine Reihe von Assoziationskomplexen aufzustellen, die aber im Grunde nichts anderes als Dünenlandschaften sind.

Wie Wald und Steppe ist auch das Moor ein Landschaftstyp. Darauf weisen manche Pflanzengeographen hin, z. B. KaTz (1941), der das Moor als die Landschaft ("Stück eines Territoriums») mit überschüssiger Feuchtigkeit definiert. Auch nach Bogdanowskaia-Guinéneuf (1946) ist das Moor eine besondere Landschaft, eine Gruppe von miteinander verbundenen spezifischen "Biocoenosen». Es ist also ein Landschaftstypus, in dem zahlreiche Pflanzenvereine unterschieden werden könøen, wie dies z. B. Oswald auf dem Moore Komosse in Schweden getan hat. Eine Aufstellung der Typen der Moorlandschaften finden wir zuerst bei CAJANDER, der schon 1904 auf den grundlegenden Unterschied in der Verteilung der Vegetation der westeuropäischen und der finnisch-lappländischen Moore hinwies. Später gab er eine Charakteristik der wichtigsten Moortypen Finnlands, des karelischen, des Aapa- und des Hümpelmoorkomplexes, zu denen AuER im Jahre 1941 den Typus des Hängemoores hinzufügte. Schließlich stellte Regel (1941) noch die Typen des Tundra-, Arktis- und Hochalpenmoors auf, die

4 Ein Fehler ist es meiner Ansicht nach, in diesem Zusammenhang von Steppenheiden zu sprechen, wie dies manche Autoren tun. Denn damit wird die Landschaft der Steppe - meist ist es aber gar keine Steppe - mit der Landschaft der Heide zusammengeworfen. Im Gebiet der Steppe gibt es nämlich keine Heiden, im Gebiet der Heiden keine Steppe, jedenfalls nicht Landschaften, die dieser entsprechen. 
alle aus bestimmten Pflanzenvereinen, aus bestimmten Kombinationen von Assoziationen bestehend charakterisiert werden können. 1925 gab Oswald eine Einteilung der Moore in Waldhochmoore, eigentliche Hochmoore, Flachhochmoore und terrainbedeckende Moore. Auch in der UdSSR entstand eine Reihe Versuche, die Moore zu klassifizieren, wie z. B. der von GaLKINA (1945), die die Moorlandschaften in vier Typen einteilte. Das Vorhandensein von so vielen Klassifikationsversuchen der Moore - nach GaLKINA sind es sechs Gruppen - und das häufige Durcheinander, das dabei herrscht, rühren davon her, da $\beta$ die meisten Pflanzengeographen und Pflanzensoziologen keinen Unterschied zwischen dem Moor an und für sich, also der Moorlandschaft, und der auf ihm vorkommenden Moorvegetation machen. Darauf weist auch ZINSERLING (1938) in seiner Beschreibung der Moore der UdSSR hin.

Schließlich können auch die Alluvionen der großen Flüsse als konkrete Landschaften aufgefaßt werden, so z. B. diejenigen, die, in drei verschiedene Horizonte (Ufer-, Mittel-, Terrassenhorizont) gegliedert, das Tal der Memel ungefähr von Kaunas bis zur Grenze des frühern Memelgebietes einnehmen. Regel hat (1930) demgemäß in Litauen einen Distrikt des Memeltales unterschieden, der in pflanzensoziologischer Hinsicht einen Komplex aus zahlreichen Assoziationen: der Wiesen, Verlandungsvereine, Gebüsche usw., also im Grunde eine Landschaft darstellt. $\mathrm{Da}$ indes der Ausdruck Alluvion in der Regel als geomorphologisch- geologischer Begriff verwendet wird, würde sich vielleicht empfehlen, statt von Alluvionen im landschaftlichen Sinne von Alluviallandschaften zu sprechen.

Als Ergebnis halten wir fest, daß Wald; Moor, Tundra, Heide, Steppe und Alluvionen nicht, wie oft behauptet, Pflanzenvereine, sondern Landschaften sind. Ihre Pflanzendecke besteht aus verschiedenen, oft physiognomisch recht verschiedenen Vereinen oder Phytocoenosen, von denen in der Landschaft «Wald» die' Lignosa, in der Landschaft "Steppe» die Duriherbosa, in der Landschaft "Heide» die Ericilignosa vorherrschen. Wälder, Moore, Wiesen, Tundren usw. sind zudem, soweit sie nicht einen ganzen Landschaftsgürtel umfassen, Geocoenosen im Sinne von Sukatschew, die in Ökotypen und Biocoenosen zerfallen. Hinsichtlich ihrer Pflanzendecke sind sie als Komplexe von Phytocoenosen aufzufassen.

Wie Landschaft und Pflanzenverein ständig verwechselt werden, so erstere und Assoziation, d. h. die Grundeinheit der Pflanzenvereine.

Du RIETz bezeichnet die Assoziation als «aus einer oder mehreren Konsoziationen (Teilassoziationen) bestehenden stabilen Phytocoenose, in der eine Schicht von einer bestimmten Gruppe von Arten mit starker soziologischer Affinität zueinander beherrscht wird, d. h. aus einer bestimmten Assozion bestehen $^{5}$... Die Zusammensetzung der übrigen Schichten vermag dagegen sehr stark zu wechseln, und auch die durchgehende Assoziation kann eine recht heterogene Artenmischung aufweisen... Wenn nichts anderes gesagt wird, so gehört die durchgehende A ssozion der obersten Schicht an.» Dieser Assoziationsbegriff, der die Vegetation allein berücksichtigt, entspricht nach Du RIETz in der Praxis dem der meisten mitteleuropäischen Pflanzensoziologen, wie RüBels, BRaun-Blanquets, Drudes, und außereuropäischer, wie Cockaynes, Nichols, Shantzs. Auch die Assoziationen und Soziationen (Teilassoziationen) der Finnen und Schweden gründen ausschließlich auf der Vegetation.

Im Gegensatz dazu stehen jene Definitionen der Assoziation, die nicht auf ihrem Pflanzenbestand allein beruhen, sondern ihren Standort miteinschließen. Hieher gehört z. B. die Formation und der Komplex mancher Autoren, wie der erwähnte Waldtyp SuKatschEws, die aber immer Landschaften entsprechen, auch wenn sie (fälschlich) als Pflanzenvereine gedeutet werden.

Es soll daher im folgenden auf die Landschafts- und Vegetationseinheiten eingetreten werden.

\section{Landschaftseinheiten und Vegetationseinheiten}

Den Begriffen Landschaft und Pflanzenverein als generellsten und abstraktesten Ausdrücken für bestimmte Erscheinungen der terrestrischen Wirklichkeit entsprechen konkrete Gebilde: Landschafts- und Pflanzenvereinsindividuen (Assoziationsindividuen), die in verschiedene Einheiten zerfallen. Eines der auffälligsten Unterscheidungsmerkmale ist die Größe, die deshalb bei Klassifikationsversuchen bisher im Vordergrund stand, ohne daß eine eindeutig-klare Gliederung erzielt werden konnte. Die Ansichten sind daher auch hierüber noch geteilt, wobei z. B. Schmid (1936) für die Biocoenose

5 Unter Assozion versteht Du RIETz eine systematische Einheit in der Phytosoziologie, die nur eine einzige Schicht (Synusie), wie z. B. die Baumschicht, die aus Kräutern und Gräsern bestehende Feldschicht, die aus Flechten und Moosen bestehende Bodenschicht, berücksichtigt. Konsoziation ist eine höhere Einheit, der Assozion übergeordnete Einheit der Synusie. In der phytosoziologischen Literatur haben die Ausdrücke Assozion und Konsozion keine weitere Verbreitung gefunden. 
betont, daß es ein aussichtsloses Beginnen sei, deren klassifikatorische Gebundenheit zu finden, bevor die allgemeine festgestellt sei.

Was die Landschaft anbelangt, erscheint sie bei Sorschalva (1947) als "ein Fragment der Erdhülle im Ganzen» und nur auf einem größeren Territorium ausgeprägt ${ }^{6}$. Seiner Auffassung fehlt die Unterteilung in kleinere Einheiten. KRüDENERs Landschaftsbegriff ist bedeutend differenzierbarer. Er nennt (1926) den Wald die kleinste natürliche Landschaftseinheit. Andere, wie PAssarge (1930), sprechen in diesem Fall allerdings von Landschaftsteilen, LAwrenko (1946) von Elementarlandschaften.

Zur Klarstellung knüpfen wir am besten an die "Naturkomplexe» von MArkus an, der darunter (1926-1936) die Gesamtheit aller Erscheinungen und Dinge versteht, die in einem gewissen Teil der Erdoberfläche lokalisiert und kausal miteinander verbunden seien. Nun besteht aber wohl kausale Verknüpfung zwischen allen Erscheinungen der Erdoberfläche, womit diese in ihrẹ Gesamtheit einen Naturkomplex darstellt. MAR Kus möchte diesen indes auf relativ kleine Einheiten beschränken, weshalb er zu den genannten Kriterien noch das der Homogenität hinzufügt. Damit sind ohne Zweifel landschaftliche Einheiten von relativ geringer Größe, Elementar- oder Mikrolandschaften, isolierbar, ohne daß Markus eindeutige Hinweise auf die möglichen Größenordnungen gibt. Doch stellt er fest, daß verschiedene Einheiten, d. h. also verschieden große Elementarlandschaften bestehen.

Die pflanzengeographische Charakteristik eines Naturkomplexes, d. h. seine Vegetation, die aus verschiedenen Pflanzenvereinen bestehen kann, repräsentiert der von vielen Pflanzengeographen, wie Oswald (1923), REGEL (1928, 1941) u. a., verwendete «Assoziationskomplex». Er wurde von Du RIETz (1932) zum Phytocoenosenkomplex ausgebaut und wie folgt definiert: Ein Phytocoenosenkomplex ist eine zusammenhängende oder in räumlich getrennte Teile zersplitterte Vegetationseinheit, die aus mit einer gewissen Regelmäßigkeit zusammengefügten Siedlungen mehrerer, vielfach gar nicht verwandter Phytocoenosen besteht. Je nach dem Grade der Komplexität unterscheidet Du RIETz die Mosaikkomplexe, die aus einem Mosaik verhältnismäßig kleiner Siedlungen, und die Zonationskomplexe, die aus mehr oder weniger zonal-angeordneten Phytocoenosen oder Mosaikkomplexen bestehen, wie es die Hochmoore, die Phytocoenosenkomplexe kleiner Inseln und Seen und der alpinen Moränenhügel sind. Weiter gehören nach Du RIETz zu den Phytocoenosenkomplexen die Vegetationsregionen und die Vegetationsstufen. REGEL wies nun (1939) darauf hin, daß seine Komplexe im Sinne von Naturkomplexen aufzufassen und landschaftliche Einheiten sind und daß die Vegetation des Komplexes nur eines seiner Merkmale oder Elemente ist, wie sich MARkus hinsichtlich der Naturkomplexe ausdrückt.

Auch die kleinste Einheit des Assoziationskomplexes kann sehr verschieden groß sein. Aber auch diese Größe hängt vom Grade der Homogenität ab. Die wenig homogene Vegetationsdecke des Hochmoores besteht aus einer Reihe Assoziationen oder Soziationen; sie ist also ein Komplex von Assoziationen und von Soziationen, aus denen auch die Vegetation der Schlenken und der Höcker besteht, die

6 Seine Schlußfolgerungen sind als Äußerung aus der russischen Wissenschaft so aufschlußreich, daß sie im folgenden wiedergegeben seien: 1. Die Landschaft ist ein Fragment der gesamten geographischen Erdoberfläche und ist nur auf größeren Gebieten vollständig entwickelt. Sie bildet eine systematische Einheit der Geographie und gleichzeitig eine Kategorie der Rayonnierung und wiederholt sich in der Regel nicht im Raume. Beispiele von Landschaften sind: die ukrainische Steppe, die südmandschurische Taiga. 2. Die Landschaften sind den Fazies untergeordnet, innerhalb deren sie miteinander durch Bindungen ersten Grades zusammenhängen. So steht z. B. die Landschaft der ukrainischen Steppe genetisch der Landschaft (Fazies) der Edellaubwälder der russischen Ebene besonders nahe. Die Vereinigung der ukrainischen Steppe mit den Steppen Westsibiriens auf zonaler Basis ist möglich, gehört jedoch nicht zu einer genetischen Rayonierung und ist auf weiter voneinander entfernten Zusammenhängen zweiten Grades, die zwischen den Landschaften bestehen, begründet. 3. Jede Landschaft enthält einen ihr eigenen qualitativen Bestand an Pflanzenassoziationen, die einem bestimmten System ökologischer Zusammenhänge unterstehen. Eine geobotanische Rayonnierung muß sich von den Merkmalen der Pflanzendecke leiten lassen, jedoch von der Landschaft ausgehen, deren Vegetation einer Einheit der geobotanischen Rayonnierung entspricht, die einen Grad höher steht, als es der geobotanische Rayon ist. 4. Die Phytocoenogenese verläuft innerhalb der Landschaft wie ein biologischer Prozeß. Die Pflanzenvereine innerhalb der Landschaft sind nicht selten vikariierende Varianten, die als erste Stufe der Absonderung phytocoenologischer Kategorien angesehen werden können. Dieses Vikariieren ist das Ergebnis einer natürlichen Auswahl, deren Verlauf durch die landschaftlichen Eigenheiten des Gebietes bestimmt wird. 5. Im Verlauf der Entwicklung und Ausbildung der Phytocoenosen treten zwei sich zum Teil widersprechende Momente auf: in genetischer Hinsicht verschiedene Elemente, die zuweilen fälschlich Konvergenzen genannt werden und Divergenzen. Das erste Moment entsteht als Ergebnis der (meist unvollständigen) Einheitlichkeit ökologischer Zusammenhänge, die sich zwischen der Umwelt und den in genetischer Hinsicht verschiedenen Komplexen der Pflanzen einstellen. (Die Entwicklung der Phytocoenosen wird durch die Umwelt [mit]bestimmt, aber das Objekt der Entwicklung ist die Vegetation selbst mit ihren biologischen Eigenschaften. Den Anfang dieser Entwicklung bestimmt das Prinzip der Divergenz, das auch die Grundlage der Systematik der Phytocoenosen und der Entwicklungstheorie ist. 6. Die Entwicklung der Phytocoenosen, die am Aufbau der Landschaft teilnehmen, ist einer der wichtigsten Faktoren der Entwicklung der Landschaft selbst. 


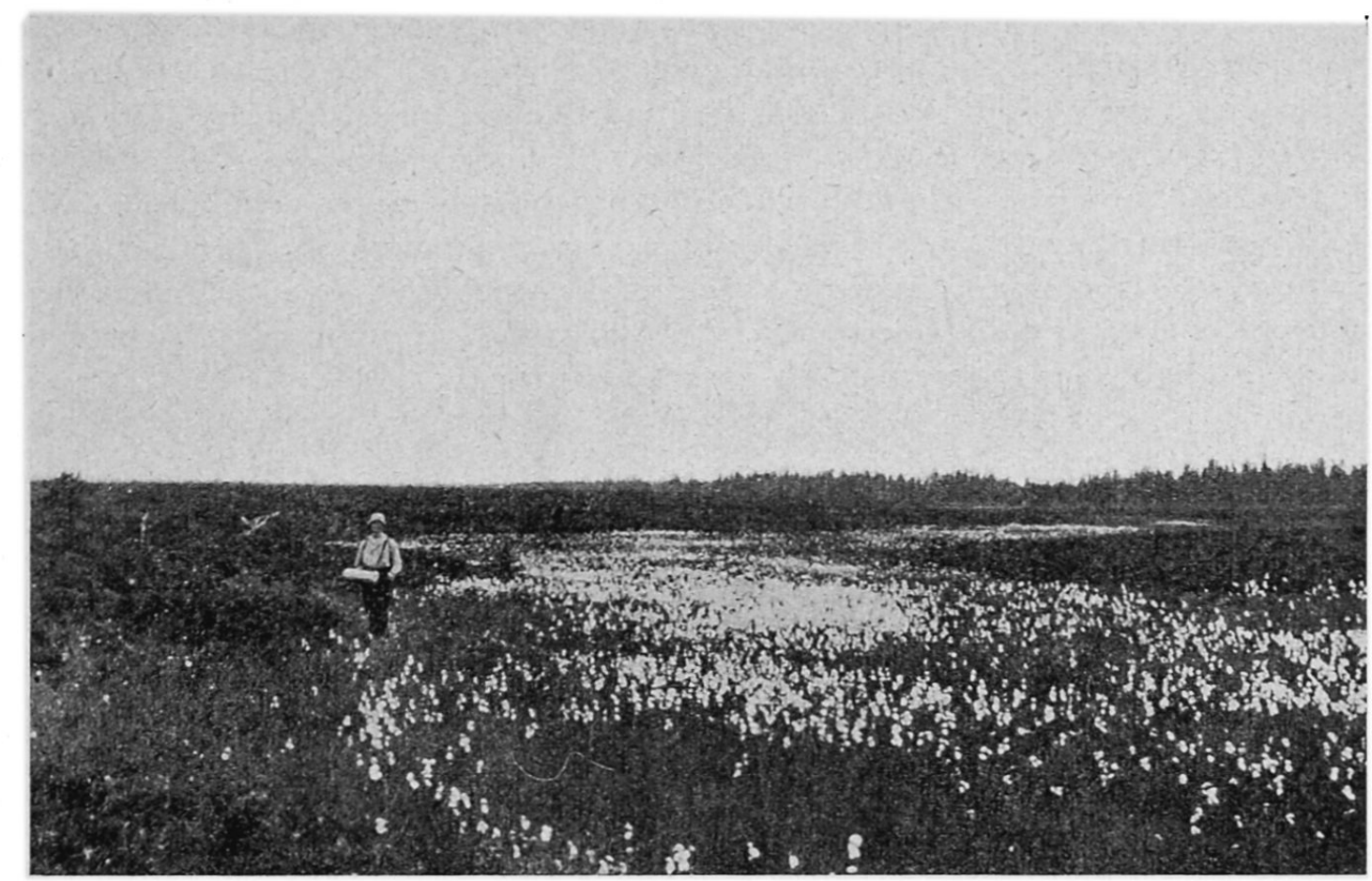

Abb. 2. Hochmoor Komosse, Schweden. Im Vordergrund aus Wollgras bestehender Verein des Grasmoors, links Moorfläche zu einem aus Torfmoosen, Sumpfporst und Zwergkiefern bestehenden Verein im Hintergrund der das Moor begrenzende moorige Wald. Ein Komplex aus verschiedenen Vereinen, die Elemente einer Moorlandschaft sind. Photo: H. Osvald. Aus Festschrift C. Schröter, Veröffentlichungen des Geobot. Instituts Rübel 1925.

ihrerseits die kleinsten landschaftlichen Einheiten der Landschaft "Hochmoor» sind. Sie wie ihre Vegetationsdecke sind homogen und damit die kleinsten Naturkomplexe. Die Höhe der Höcker variiert je nach Umwelt zwischen wenigen Zentimetern und mehreren Metern (an der polaren Waldgrenze z. B. sind die Torfhümpel bis zu $2 \mathrm{~m}$ hoch). Ähnliche Größenmaße weist der Horizontaldurchmesser der Höcker und der Schlenken auf. Folgende Worte von Markus erläutern das hier grundsätzlich Gesagte: «die Naturkomplexe stellen Gruppen gemeinschaftlich auftretender kleinerer Naturkomplexe dar. Nur die kleinsten homogenen Landschaften können nicht in noch kleinere Landschaften aufgeteilt werden.»

Diese kleineren Einheiten würden zum Teil den Biocoenosen entsprechen, die DäNIKER (1939) jedoch als Einheiten der Vegetation ansieht. Die Biocoenosen werden aber sowohl durch Tiere als auch durch Pflanzen, also durch Lebewesen charakterisiert. Ein Teil davon ist nach LAwrenko (1945) die nur aus Pflanzen bestehende Phytocoenose. Die Biocoenose bildet nach ihm einen Teil eines noch komplizierteren Ganzen, der Elementarlandschaft, oder, wie sie Sukarschew (1942) zu bennen vorschlägt, der Geocoenose. Diese Geocoenose zerfällt in die Biocoenose und den Oekotypus, welch letzterer Name von Wysotzki stammt und worunter die Gesamtheit aller klimatischen und edaphischen Faktoren verstanden wird. Zum Begriff der Phytocoenose gehören nach Lawrenko die Standortsfaktoren nicht. "Die Phytocoenose und die Geocoenose», sagt Sukarschew mit Recht, "sind verschiedene Begriffe, die in ihrer Entwicklung verschiedenen Gesetzmäßigkeiten unterworfen sind, jedoch in gegenseitiger Beziehung stehen.»

Wir kommen so zu folgender Schlußfolgerung: Die Elementarlandschaft, der Naturkomplex nach Markus, oder die Geocoenose Sukatschews zerfällt je nach den Merkmalen, deren wir uns zu ihrer Charakterisierung bedienen, in folgende Teile, in den Oekotypus, die Gesamtheit aller klimatischen und edaphischen Merkmale des Reliefs der Erdoberfläche; in die Biocoe nose, die Gesamtheit aller lebenden Wesen (ein zuerst von GAMs [1918] im gegenwärtigen Umfang gebrauchter Ausdruck, mit Phyto- und Zoocoenose, d. h. die pflanzlichen und tierischen Bewohner einer bestimmten Landschaft, wozu in den meisten Gebieten der Erde noch die Anthropocoenosen, d. h. die menschlichen Siedlungen, kommen) und (gegebenenfalls) in den wirtschaftlichen Rayon, also die Gesamtheit aller wirtschaftlichen Erscheinungen, soweit diese für die Charakterisierung der Landschaft von Bedeutung sind. 
Immer handelt es sich dabei um Vergesellschaftungen von anorganischen und organischen Erscheinungen, um "Gesamteffekte der Korrelation Litho-, Hydro-, Atmound Biosphäre der Erde» (WINkLER), während Phytocoenosen rein botanische Naturobjekte sind. Die Notwendigkeit einer klaren Unterscheidung von Landschaft und Pflanzenverein, bzw. von geographischen und botanischen Untersuchungsgegenständen besteht demgemäß auch — was im Grunde selbstverständlich ist - hinsichtlich ihrer verschiedenen Einheiten. Indes ist gerade auf diesem Gebiet sowohl von der Pflanzensoziologie als auch von der Geographie als Landschaftsforschung noch so gut wie alles zu tun (vgl. z. B. HARTShorne, 1946, und SChMithüsen, 1948).

\section{Zur Frage der Realität der Assoziation}

In der pflanzensoziologischen Literatur wird immer wieder diskutiert, ob die grundlegende Einheit der Pflanzengesellschaften, die Assoziation, eine Realität sei. Die einen, wie früher der Verfasser und eine Reihe der bekanntesten Pflanzengeographen und Pflanzensoziologen mit SChröter (1902), Braun-Blanquet (1932) u. a., sagen, die Pflanzenassoziation sei, ebenso wie die Art in der Systematik, eine Abstraktion. Andere, darunter ebenfalls eine Reihe der bekanntesten Autoren mit DU RIETZ (1921) und AleChIN (1926), behandeln sie als Realität, und dritte endlich betonen, sie sei Realität und Abstraktion zugleich.

Diese Auffassungsdifferenzen gründen kaum in Beobachtungsfehlern; denn wir haben es mit durchwegs guten Beobachtern zu tun. Zum Teil beruhen sie auf unzulänglicher begrifflicher Umformung von Beobachtungen, so wenn auf Grund von Probeflächenuntersuchungen direkt die abstrakte Assoziation, d. h. der Typus, gebildet wird. Zum andern gehen sie aber wohl auch auf die Vermengung von Landschaft und Pflanzenverein zurück, die ja an sich bereits bei der Bildung der obersten Begriffe zu Unklarheiten geführt hat, wie dargestellt wurde, und bei der Aufstellung von Einheiten begreiflicherweise zu analogen Kollisionen Anlaß geben muß. Untersuchen nämlich die Pflanzensoziologen einen Pflanzenverein, so beziehen sie in ihre Arbeit meist nicht nur dessen Pflanzenbestand, sondern - mit einem gewissen Recht - auch dessen. Umwelt ein, womit dann freilich eine Landschaft erfaßt wird.

Das Gesagte sei am Beispiel des Piceetum myrtillosum, des an Schwarzbeeren reichen Fichtenwaldes, eines der verbreitetsten "Pflanzenvereine» des nördlichen Europas und der Alpen, erläutert. Dieser ist zweifellos eine Landschaft, d. h. eine Einheit von bestimmten Pflanzen, Böden, Klimaten usw. Deren Pflanzendecke, d. h. dem. Piceetum myrtillosum im engern Sinn, sind oft, namentlich in Gebirgsgegenden, fleckenartig andere Vereine eingelagert, die durch Relief- und Bodenunterschiede erklärbar erscheinen. Für die Landschaftsbeschreibung dieses Waldtyps sind sie kaum von Belang, bei der Fixierung der Assoziation jedoch müssen sie ausgeschieden werden. M. a. W. man abstrahiert auf Grund mehrerer Vegetationsbeschreibungen das "reine» Piceetum myrtillosum, das in der Natur möglicherweise überhaupt fehlt; d. h. man stellt Typen desselben, z. B. das Piceetum myrtillosum polare, boreale oder alpinum, auf. Sie alle sind als Assoziationen Abstraktionen, die in der Natur untersuchten jedoch sind Landschaften und Realitäten. Die Frage besteht nun allerdings, ob ein reales Piceetum myrtillosum als Pflanzenverein dennoch denkbar sei. Doch scheint mir dies kaum möglich, da mit dessen Realität die Attribute der Umwelt so eng verbunden sind, daß in der Praxis diese mit der Assoziation stets konsequent zusammen behandelt werden.

Was die noch nicht erörterte Möglichkeit der Aufstellung von Landschaftstypen eines landschaft lichen Piceetum myrtillosum z. B., anbetrifft, so brauche ich nur auf $C_{A J A N D E R S}$ Myrtillus-Typus oder Sukatschews Piceetum myrtillosum hinzuweisen, die Landschaften sind, auch wenn sie fälschlich Pflanzenvereine genannt werden.

$\mathrm{Zu}$ diesen Ansichten kam der Verfasser u. a. während einer Tagung der fennobaltischen Pflanzengeographen auf den Alandsinseln 1938. Unter Leitung A. PaLmGrens wurden die Laubwiesen besichtigt, wobei sich auf den einzelnen Inseln immer neue Arten einstellten, so daß diese sich eigentlich als Wiesen-Landschaften erwiesen. Denn ihr Pflanzenbestand entsprach verschiedenen Wiesen-Assoziationen, besser Herbosae (RüBEL), zu denen noch Bäume und Sträucher traten. Die Darstellung von PALMGREN (1915) über deren Zusammensetzung macht eindrücklich genug, wie mannigfaltig ihre Vegetation ist. Doch sind die von ihm aufgestellten Sesleria-Wiesen nicht Realitäten, sondern Abstraktionen, d. h. Beschreibungen ausschließlich ihrer Vegetation, nicht ihrer konkreten Landschaft, im Gegensatz zu den Laubwiesen, die Landschaften sind.

Grundsätzlich wäre die Lösung der ganzen Frage insofern einfach, als jedes Gebilde der Wirklichkeit abstrakt und konkret zugleich, d. h. als Individuum und Typus, aufgefaßt werden könnte. Dies gälte somit von der Assoziation wie von der Landschaft; 
doch vertritt der Verfasser die Ansicht, daß erstere ausschließlich Abstraktion ist, während für das konkrete Komplementärgebilde weder Name noch Vorstellung besteht.

\section{Klassifikationsprobleme}

Es ist klar, daß die Vermengung der Begriffe Landschaft und Pflanzenverein sich auch auf deren Systematik auswirkt und damit sogar als ein Grund dafür betrachtet werden mag, daß diese bisher nicht wesentliche Ergebnisse zu erzielen vermochte. Dabei darf wohl als Selbstverständlichkeit betrachtet werden, daß jede Klassifikation, wenn sie sich als "natürlich» ausgeben will, auf der Gesamtheit aller Merkmale des zu klassifizierenden Gegenstandes aufzubauen hat. Zumal sollte dieser Grundsatz in der biologischen Wissenschaft' und damit in der Pflanzensoziologie Gültigkeit beanspruchen können; die doch auf eine lange und instruktive Geschichte der Systematik zurückzublicken vermag. In Wirklichkeit steht jedoch gerade die Pflanzensoziologie noch in der Phase vorwiegend «künstlicher», d. h. partieller, auf einzelnen Elementen der Pflanzenvereine basierender Systematiken.

Es dürfte einleuchten, daß zur Klassifikation der Phytocoenosen ausschließlich botanische Merkmale: Physiognomie, soziale Struktur, Treue, Konstanz, floristische $\mathrm{Zu}-$ sammensetzung, Deckungsgrad, Abundanz, Soziabilität, ökologische Valenz (Vouk, 1938) heranzuziehen sind, wie BraUn-Blanquet (1921) zeigte, der die soziologische Progression in den Vordergrund stellte, während geographische Objekte mittels landschaftlichen Merkmalen gegliedert werden müssen. Bisher wurden indes hauptsächlich künstliche, auf einem einzigen oder wenigen Merkmalen aufgebaute Systeme entwickelt.

Vor allem ist unter ihnen das bereits eingangs erwähnte, von BROCKMANN-JEROSCH und RÜBEL aufgestellte und dann von RüBEL durchgeführte zu nennen, das an sich jedoch seinen Zweck durchaus erfüllt, da das ihm zugrunde gelegte Klassifikationsmoment, die Physiognomie der Vereine, den Ausdruck der Gesamtheit der Milieubedingungen wie der Ausbildungsmerkmale darstellt, wobei es allerdings, wie gleichfalls schon betont, nicht konsequent gehandhabt wurde. Eine andere, auf den "Charakterarten" fußende Klassifikation der Pflanzenvereine stammt von BRAUN-BLANQUET, der sie auch in zahlreichen Arbeiten anwendete. Sie jedoch ist nicht nur rein deduktiv, künstlich, sondern auch umstritten, da die Bedeutung der Charakterart für den Pflanzenverein von den Pflanzensoziologen (LüDr 1928, Du RIETz 1932 u. a.) verschieden beurteilt wird. Bei der Verwendung dieses Merkmals werden ganz verschiedene Gruppen von Vereinen zusammengefaßt; es wird zugleich eine Hierarchie von Klassen, Ordnungen usw. aufgestellt, die, ähnlich der Linnéschen Heterogenes vereinigt, Verwandtes trennt, nur weil konsequent das eine Merkmal - wie z. B. bei LINNÉ die Zahl der Staubgefäße - benutzt ist. Die Braun-Blanquetsche Einteilung der Pflanzenvereine beruht übrigens auf der schon 1909 von Gradmann geäußerten Ansicht, daß die floristische Methode die einzige sei, die sich vollständig durchführen lasse. Doch müsse, sagte dieser, bei ihrer Anwendung der gesamte Artenbestand berücksichtigt werden, und er warnte auch davor, sich von vornherein auf eine bestimmte Einteilung festzulegen und die "Einzelformationen in ein Prokustesbett» zu spannen. GradmanN war also auf dem richtigen Wege, nur fehlte ihm die genauere Kenntnis vom Wesen der Pflanzenvereine. Das von ihm geforderte und befolgte «induktive Vorgehen» ist im übrigen nur so möglich, daß verschiedene soziologische Kriterien, nicht nur der Artenbestand, zur Klassifikation verwendet werden. Was hierbei die Charakterarten anbelangt, so eignen sie sich nur für die Kennzeichnung kleiner lokaler Phytocoenosen, während für größere Einheiten andere Momente, wie in erster Linie wohl die Physiognomik und dann erst floristische Zusammensetzung, soziale Struktur usw. heranzuziehen sind.

Im ganzen ist festzustellen, da $\beta$ nur ein umfassendes Studium aller Merkmale der Pflanzenvereine uns zu einem natürlichen System führt, eine Arbeit, die erst noch zu leisten ist, analog übrigens der Landschaftscharakterisierung, deren klassifikatorische Erfassung ebenfalls in den Anfängen steht. Diese, Generationen erfordernde Arbeit zu leisten, konnte hier nicht beabsichtigt werden.

\section{Unsere Erörterung läßt sich in folgende Leitsätze zusammenfassen:}

Pflanzenverein und Landschaft sind streng auseinanderzuhalten, wenn Irrtümer, Ungenauigkeiten und Mißerfolge in Definition, Klassifikation und Verwendung beider vermieden werden sollen. - Im Unterschied zur Landschaft, die von den Geographen 


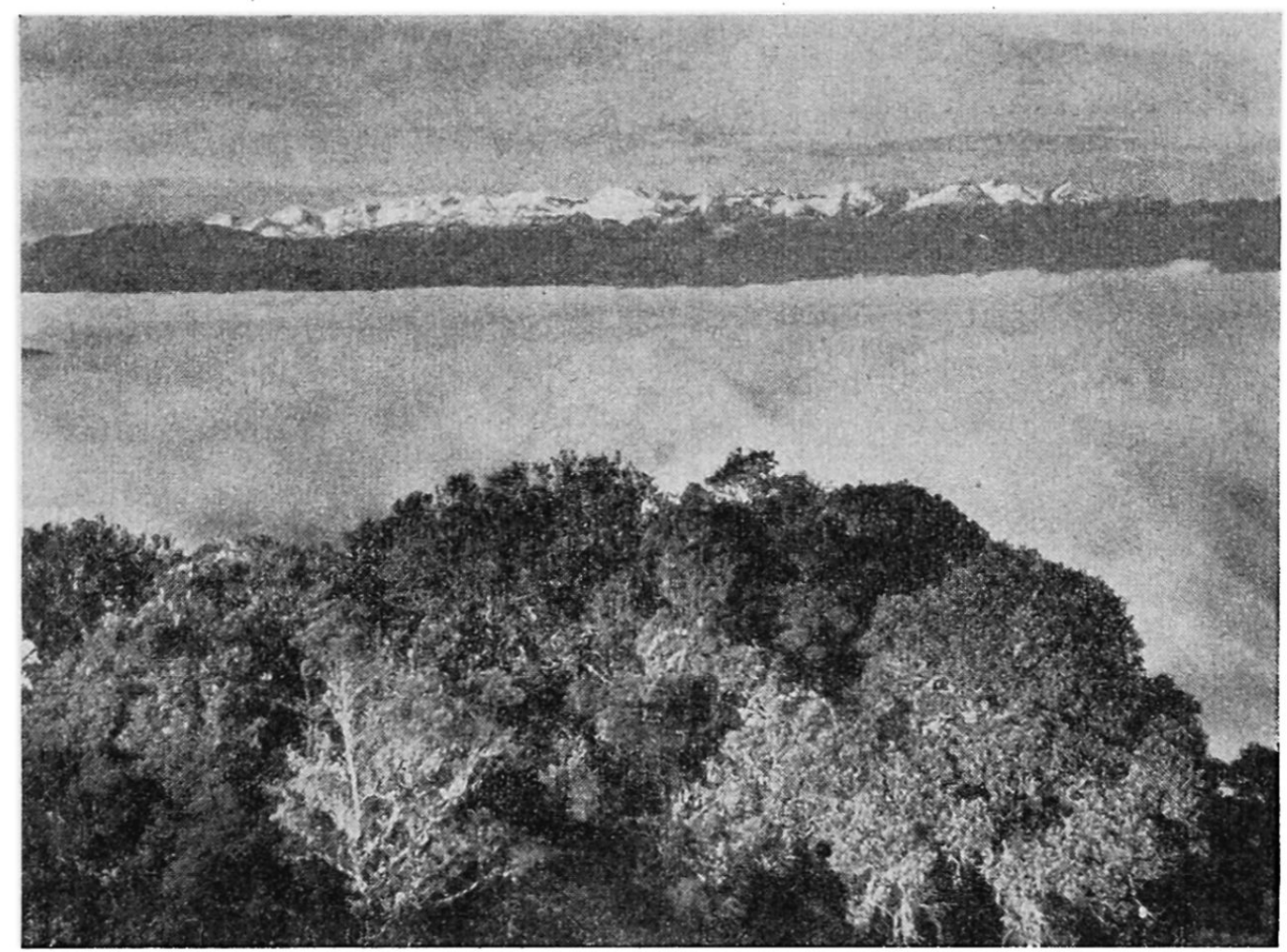

Abb. 3. Tropischer Höhen- und Nebelwald am Ostabfall der bolivianischen Hochkordillere, Provinz Inquisivi. Im Hintergrund Cordillera Quimzacruz. Hochgebirgswaldlandschaft an der Waldobergrenze $(3600 \mathrm{~m})$ als Beispiel des Unterschieds zwischen Wald als Landschaft und bloßen Gehölzformationen. Photo: C. Troll. Aus Bericht über das Geobotanische Forschungsinstitut Rübel, für das Jahr 1947. Zürich 1948.

mehr oder minder anerkannt ein bestimmtes Stück der Erdhülle, das hinsichtlich Boden, Klima, Gewässer, Tier- und Pflanzenwelt, Mensch- und Menschenwerk ein (harmonisches) Ganzes bildet, also durch zahlreiche sachlich verschiedene Merkmale charakterisiert ist, repräsentiert die Pflanzendecke ein ausschließlich botanisches, also biologisches Gebilde und ist als solches im Rahmen der Landschaft lediglich Teilobjekt. Sie ist freilich meist ein charakteristisches Merkmal der Landschaft. Der Pflanzenverein, die Phytocoenose, ist die botanische Charakteristik einer bestimmten Landschaftseinheit, die pflanzengeographische Provinz die botanische Charakteristik einer größern landschaftlichen Einheit, einer geographischen Provinz. - Die kleinste landschaftliche Einheit ist die Elementarlandschaft, die Geocoenose, der Naturkomplex. Sie zerfällt in Oekotypus, Biocoenose und (eventuell) in den anthropogenen, wirtschaftlichen Rayon. Die Biocoenose zerfällt in Phyto-, Zoo- und Anthropocoenosen. Wald, Moor, Heide, Steppen, Tundren usw. sind keine Pflanzenvereine, sondern Landschaften, die aus verschiedenen Pflanzenvereinen bestehen. Assoziation wie Soziation dürfen nur als Phytocoenosen, nicht als Landschaften aufgefaßt werden, daher ist zu ihrer Charakteristik der Standort nicht benutzbar. - Die Assoziation kann - wie übrigens auch die Landschaft - sowohl als Abstraktion wie als konkretes Gebilde betrachtet werden. Im ersten Fall handelt es sich um Vegetationstypen und Normen, im letztern um Individuen. Gegenwärtig, besteht Assoziation im pflanzengeographisch-soziologischen Sinne nur als Abstraktum, als Typusbegriff. Für das komplementäre konkrete Gebilde fehlt noch Name wie Vorstellung. - Die natürliche Klassifikation der Pflanzenvereine wie Landschaften muß auf Grund aller ihrer Merkmale erfolgen. Eine nur auf den physiognomischen Merkmalen oder auf den Charakterarten aufbauende Klassifikation ist künstlich. 
Literatur. Die mit einem * bezeichneten Titel sind russisch, vom Verfasser jedoch übersetzt, während die übrigen russischen Arbeiten bereirs im Original mit einer Übersetzung in eine fremde Sprache versehen sind. Diese russischen Studien enthalten jedoch keine fremdsprachigen Zusammenfassungen. - Alechin, W.: Assoziationskomplexe und Bildung ökologischer Assoziationsreihen. Jahrbücher für systematische Botanik, 59, 1924. Ders.: Was ist eine Pflanzengesellschaft? Repertorium specierum regni vegetabilis, Beiheft XXXVII, 1926. Ders.: Ist die Pflanzenassoziation eine Abstraktion oder eine Realität? Botanische Jahrbücher, 60, 1926. - AUER, V.: Moorforschungen in den Vaaragebieten von Kuusamo und Kuolajärvi. Communicationes ex Instituto quaestionum forestalium Finlandiae editae, 16, 1923. - BERG, L.: Geographical Zones of the USSR. I. Tundra. Forest-zone. Leningrad 1930. Supplement Nr. 42 zu Bulletin of Applied Botany, Genetics and Plant Breeding, 1930. Braun-Nlanouet, J.: Prinzipien der Systematik der Pflanzengesellschaften auf floristischer Grundlage. Jahrbücher der St. Gallischen Naturwissenschaftlichen Gesellschaft, 57, II, 1921. Ders.: Plant Sociology. New York and London 1932. - Brockmann-JerosCh, H., und RüBeL: Die Einteilung der Pflanzengesellschafcen nach ökologisch-physiognomischen Gesichtspunkten. Leipzig 1912. — *BoGDanowskaja-Guinéneuf, J.: Die Pflanzendecke der Hochmoore des russischen Baltikums. Arbeiten des Peterhofer Naturwissenschaftlichen Forschungsinstitutes, Nr. 5, 1928. Russisch. Dies.: Sur quelques questions fondamentales de la paludologie. Journal botanique de l'U.R.S.S. XXXI, Nr. 2, 1946. Russisch. - ${ }^{*}$ Busch, N.: Pflanzengeographische Skizze des europäischen Teils der UdSSR und des Kaukasus. Moşkau und Leningrad 1936. Russisch. - CAyANDER, A.: Ein Beitrag zur Entwicklungsgeschichte der nordfinnischen Moore. Fennia 20, Nr. 7, 1904. Ders.: Úber Waldtypen. Acta forestalia. Fennica 1 und Fennia 28, 1909. Ders.: Studien über die Moore Finnlands. Acta forestalia. Fennica 2, 1913. - DÄNıKER, A.: Die Biocoenose als Einheit der Vegetation. Verhandlungen der Schweizerischen Naturforschenden Gesellschaft 1939. - Flahault, C., und SChrörer, C.: Phytogeographische Nomenklatur. Zürich 1910. - *DAroshKin, I., und Rawdo: Rayon-Charakteristik der Erkrankung der Kartoffel in der BSSR. Minsk. Weißrussisch. - Du RIErz, E.: Zur methodologischen Grundlage der modernen Pflanzensoziologie. Wien 1921. Ders.: Vegetationsforschung auf sozialanalytischer Grundlage. Handbuch der biologischen Arbeitsmethoden, Abt. XI, Teil 5. Pflanzengeographische Ökologie. - Berlin und Wien 1932. - Galkina, E.: Swamp Landscapes and Principles of their Classification. Volume of Scientific Works Komarow Botanical Institute. Leningrad 1946. Russisch. - GAMs, H.: Prinzipienfragen der Vegetationsforschung. Zürich 1918. - *GorodKow, B.: Die Vegetation der Tundrazone der UdSSR. Moskau und Leningrad 1935. Russisch. - GradmanN, R.: Über Begriffsbildung in der Lehre von den Pflanzenformationen. Botanische Jahrbücher für Systematik, 43, 1909. Ders.: Wüste und Steppe. Geographische Zeitschrift, 22, 1916. - HARrshorne, R.: The Nature of Geography. Annals of the Association of American Geographers. Lancaster 1939. 2. Auflage. 1946. Katz, N.: Sümpfe und Moore. Moskau 1941. Russisch. - Keller, B.: Die Pflanzenwelt der russischen Steppen, Halbwüsten und Wüsten. Ökologische und phytosoziologische Studien, Lieferung 1. Woronesch 1923. Russisch. - Korchagin, A.: On the Principles of Classification of Forest Groupings. Volume of Scientific Works. Komarow Botanical Institute. Leningrad 1946. Russisch. - *KoRSHINSki, H.: Die Steppen. Enzyklopädisches Wörterbuch von Brockhaus-Jefron, 31 A. St. Petersburg 1901. Russisch. - * KRAsNow, A.: Die Grassteppen der nördlichen Halbkugel. Moskau 1894. Russisch. - * KrüDENER, A.: Grundzüge der Klassifikation der Waldtypen und deren volkswirtschaftliche Bedeutung im Landeshaushalt. Materialien zur Kenntnis des russischen Waldes, I. Petrograd 1916. Russisch. Ders.: Waldtypen als kleinste natïrliche Landschaftseinheiten bzw. Mikrolandschaftstypen. Petermanns Geographische Mitteilungen, 72, 1926. - Lautensach, H.: Úber die Erfassung und Abgrenzung von Landschaftsräumen. Comptes rendus du Congrès International de Géographie, II. Amsterdam 1938. - Lawrenko, E.: Sowjet-Geobotanik (Phytocoenologie). Advances of the Biological Sciences in the USSR within the recent 25 years 1917-1942. Moskau und Leningrad 1946. Russisch. Lüdr, W.: Der Assoziationsbegriff in der Pflanzensoziologie. Bibliotheca botanica, 96, 1928. - MARKus, E.: Das Komplexprofil von Jätasoo. Sitzungsberichte der Naturforscher-Gesellschaft in Tartu, 32. 1925. Ders.: Naturkomplexe. Sitzungsberichte der Naturforscher-Gesellschaft in Tartu, 32, 1926. Ders.: Verschiebung der Naturkomplexe in Europa. Geographische Zeitschrift, 33, 1926. Ders.: Die Grenzverschiebung des Waldes und des Moores in Alatskivi. Acta et Commentationes Universitatis Tartuensis, A XIV, 1929. Ders.: Naturkomplexe in Alatskivi. Acta et Commentationes Universitatis Tartuensis, Y XVIII-8, 1930. Ders.: Die südöstliche Moorbucht von Lauge. Acta et Commentationes Universitatis Tartuensis, A XXIV-5, 1933. Ders.: Geographische Kausalität. Tartu 1936. - Morozow, G.: Die Lehre vom Walde. Neudamm 1928. - Muris, O.: Der neue Landschaftsbegriff in der erdkundlichen Wissenschaft. Die Volksschule 26, 1930. - Oswald, H.: Die Vegetation des 
Hochmoores Komosse. Uppsala 1931. Ders.: Die Hochmoortypen Europas. Festschrift Carl Schröter. Veröffentlichungen des Geobotanischen Institutes Rübel in Zürich, 3, 1925. - PALMGREN, A.: Studier öfver löfängsomradene pa Aland. Acta Societatis pro Frauna et Flora. Fenicca 42, 1915. - PAsSARGE, S.: Die Grundlagen der Landschaftskunde, I. Hamburg 1916. Ders.: Wesen, Aufgaben und Grenzen der Landschaftskunde. Petermanns Geographische Mitteilungen, Ergänzungsheft Nr. 209, Gotha 1930. - Penck, A.: Morphologie der Erdoberfläche. Stuttgart 1894. - REgel, C.: Zur Kenntnis des Baumwuchses an der polaren Waldgrenze. Sitzungsberichte der Naturforschenden Gesellschaft in Dorpat, XXIV (1915), 1921. Ders.: Statistische und physiognomische Studien an Wiesen. Acta et Commentationes Universitatis Dorpatensis, A I-4, 1921. Ders.: Zur Klassifikation der Assoziationen der Sandböden. Botanische Jahrbücher für Systematik, 61, 1928. Ders.: Lietuvos pasiskirstymas i augalu geografijos dalis-Kosmus (Kaunas). 1930. Ders.: Pflanzensoziologische Studien aus dem nördlichen Rußland. III. Reliktenvereine in der Arktis. Die Moore von Nowaja Semlja. Beiträge zur Biologie der Pflanzen, 23, 1933. Ders.: Geobotanische Beobachtungen auf einer Reise in Marokko und in der Schweiz. Ergebnisse der Internationalen Geobotanischen Exkursion in Marokko und Westalgerien. Veröffentlichungen des Geobotanischen Institutes Rübel in Zürich, 11, 1939. Ders.: Komplexe, Landschaft, Vegetationsprovinz. Verhandlungen der Schweizerischen Naturforschenden Gesellschaft. 1939. Ders.: Die Vegetationsverhältnisse der Halbinsel Kola. Repertorium speciorum novarum regni vegetabilis, Beiheft LXXXII, 1941. - RüBEL, E.: Pflanzengesellschaften der Erde. Bern 1930. Schmid, E.: Was ist eine Pflanzengesellschaft. Berichte der Schweizerischen Botanischęn Gesellschaft, Festband Rübel, 46, 1936. - SCHMrrhüsEN, J.: Vegetationsforschung und ökologische Standortslehre in ihrer Bedeutung für die Geographie der Kulturlandschaft. Zeitschrift der Gesellschaft für Erdkunde zu Berlin, 1942. Ders.: Fliesengefüge der Landschaft und Ökotop. Berichte zur Deutschen Landeskunde, 5, 1948. - Schröter, C., und Kirchiner, O.: Die Vegetation des Untersees. Lindau 1902. *Semjonow-Tjanschanski, W.: Rayon und Land. Leningrad 1928. Russisch. - Sotschawa, W.: Landschaft und Phytocoenose. Referate der wissenschaftlichen Forschungsarbeiten für 1945. Biologische Wissenschaften. A kademie der Wissenschaften der UdSSR, Moskau und Leningrad 1947. Russisch. - * Sukatschew, W.: Anleitung zur Untersuchung der Waldtypen. Leningrad 1931. Russisch. Ders.: Die Untersuchung der Waldtypen des osteuropäischen Flachlandes. Handbuch der biologischen Arbeitsmethoden, Abt. XI, Pflanzengeographische Ökologie, 2. Hälfte. Berlin und Wien,1932. Ders.: Dendrologie. Leningrad 1932. Russisch. Ders.: Die Idee der Entwicklung in der Phytocoenologie. Sowjet-Botanik, 1942,1-3. Russisch. Zitiert nach Korchagrn. 1946. - Vouk, V.: Thermal Vegetation and Oecological Valence Theory. Hydrobiologia, 1, 1948. - WInkLER, E.: Grundsätzliches. Schweizer Geograph, 10, 1933. Ders.: Raumbegriff in der Geographie. Petermanns Geographische Mitteilungen, 83, 1937. Ders.: Das System der Geographie und die Dezimalklassifikation. Geographica Helvetica, 1, 1946. - *Zinserling, J.: Die Geographie der Pflanzendecke des nordöstlichen Teils der UdSSR. Leningrad 1934. Russisch. Ders.: Die Pflanzendecke der Moore. Vegetatio UdSSR. Moskau und Leningrad 1938. Russisch.

\section{PAYSAGE ET ASSOCIATION DE PLANTES}

Dans la sociologie des plantes, les définitions "paysage» et "association de plantes» sont souvent confondues voire utilisées identiquement. Cet état de fait conduisant à une situation embrouillée et contradictoire n'est à surpasser que par une séparation nette des deux termes. L'association des plantes est une conception uniquement botanique ne comprenant que des phénomènes botaniques, et le paysage, notion purement géographique, utilise les faits botaniques comme simples éléments. De nombreux exemples, extraits notamment de la littérature scientifique russe, tâchent de le prouver.

\section{PAESAGGIO E ASSOCIAZIONE VEGETALE}

Nella sociologia vegetale vengono spesso scambiati i due concetti paesaggio e associazione vegetale, determinando immancabilmente confusioni e controsensi. La situazione può essere superata se si attribuiscono valori ben delimitati ai due concetti: associazione vegetale dovrebbe restare un concetto puramente botanico (comprendente solo fenomeni botanici) e paesaggio dovrebbe di contro ricevere un valore esclusivamente geografico (in quanto i fenomeni botanici sono solo elementi del concetto). L'autore giustifica il suo ragionamento appoggiandosi a una documentazione tolta sopratutto dalla letteratura scientifica russa. 